\title{
Breeding protocol for the sand fly Nyssomyia neivai (Diptera: Psychodidae) in laboratory conditions
}

\author{
Thais Marchi Goulart ${ }^{\mathrm{a}}$, Flávia Benini da Rocha Silva ${ }^{\mathrm{b}}$, Vicente Estevam Machado ${ }^{\mathrm{b}}$, \\ Mara Cristina Pinto ${ }^{\mathrm{b}, *}$ \\ a Departamento de Biologia Animal, Universidade Estadual de Campinas, Campinas, SP, Brazil \\ ${ }^{\mathrm{b}}$ Universidade Estadual Paulista (UNESP), Faculdade de Ciências Farmacêuticas, Câmpus Araraquara, Araraquara, SP, Brazil

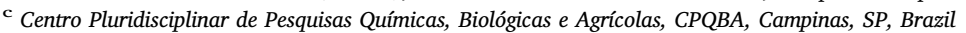
Wanderson Henrique Cruz Oliveira ${ }^{\mathrm{b}}$, Camila Feitosa de Castro ${ }^{\mathrm{b}}$, Marili Villa Nova Rodrigues ${ }^{\mathrm{c}}$,

\section{A R T I C L E I N F O}

\section{Keywords:}

Phlebotomine

Sand flies

Colony

Colonization

Life cycle

Rearing methods

\begin{abstract}
A B S T R A C T
The information in this protocol covers from the basic steps and material necessary to start a sand fly colony up to the specific details which are important to the success of a Nyssomyia neivai colony. The greatest problems in our colony of $\mathrm{Ny}$. neivai were solved with specific care, for instance, using vermiculite and an adequate number of adults in oviposition containers; the control of fungus with the exact amount of diet for the larvae and humidity control; a second blood meal for females and control of the number of times animals are used for blood meals. Currently, our colony is at F22 generation.
\end{abstract}

\section{Introduction}

The present protocol is the result of many attempts to start and maintain a colony of Nyssomyia neivai, a vector of cutaneous leishmaniasis in South America. Some scientific results which support this protocol have already been published (Goulart et al., 2015). However, we intend to help sand fly researchers by providing general and basic information on the routines that may be useful for any sand fly species and, more specifically, details of $\mathrm{Ny}$. neivai which allowed the success of our colony.

Observation is the key to the success of the colony and must be undertaken continuously. Without effort and persistence, the colony will not be successful. Probably, this is one of the reasons why, despite the great epidemiological interest in this group of insects and the relevance of colonies for research, less than $10 \%$ of sand fly species have been colonized in the laboratory (Volf and Volfova, 2011).

Since the seminal works of Barretto (1942) and Killick-Kendrick and Killick-Kendrick (1991) up to Meneses et al. (2014), using digital media, the efforts of many authors have contributed to improvement in the knowledge on different sand fly species colonization. We highlight the studies with Dampfomyia anthophora, Lutzomyia cruciata, Psathyromyia shannoni, Micropygomyia vexator (Young et al., 1981); Phlebotomus ariasi (Killick-Kendrick and Killick-Kendrick, 1987); Ph. papatasi and Lu. longipalpis (Modi and Tesh, 1983); Nyssomyia intermedia (Rangel et al., 1985; Rangel et al., 1986); Lu. longipalpis (Elnaiem and Ward, 1991; Elnaiem and Ward, 1992; Souza et al., 1999); Ph. papatasi, Ph. argentipes, Ph. duboscqi and Lu. longipalpis (Lawyer et al., 1991); Migonemyia migonei (Nieves et al., 1997); Ny. intermedia and Lu. longipalpis (Wermelinger and Zanuncio, 2001); Pintomyia serrana (Santamaría et al., 2002); Ny. intermedia and Ny. neivai (Andrade Filho et al., 2004); and $P h$. papatasi, Ph. duboscqi, Ph. sergenti, Ph. perniciosus, Ph. tobbi, Ph. halepensis, Ph. arabicus, Ph. argentipes and Lu. longipalpis (Volf and Volfova, 2011).

\section{Material and methods}

\subsection{Collection of sand flies}

The starting point of the colony is an expressive number of sand flies collected from the field, allowing production of large quantities of eggs in the laboratory. Collection of insects can be performed using Castro aspirators, Shannon and/or light traps. Collected insects must be placed into a previously prepared voile cage $(30 \times 30 \times 30 \mathrm{~cm})$. The cage is wrapped in a transparent plastic bag, and two Petri dishes are placed: one directly above the voile of the cage ceiling and the other on the bottom of the plastic bag. Cotton soaked in water to maintain high

\footnotetext{
*Corresponding author at: Faculdade de Ciências Farmacêuticas, UNESP - Universidade Estadual Paulista, Araraquara, Departamento de Ciências Biológicas, Laboratório de Parasitologia, Rodovia Araraquara-Jaú km 01, 14801-902, Araraquara, SP, Brazil.

E-mail address: marap@fcfar.unesp.br (M.C. Pinto).
} 


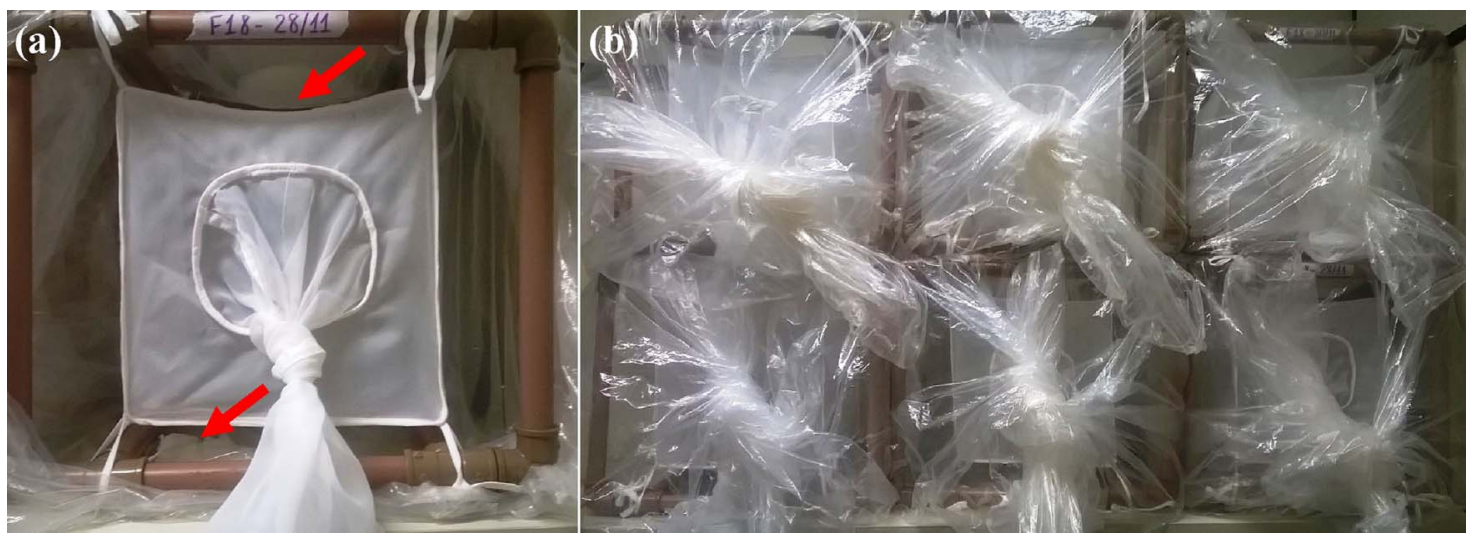

Fig. 1. Cages for the maintenance of insects. (a) Petri dishes (arrows) inside the bag, (b) Plastic bags wrapping the cages to maintain humidity.

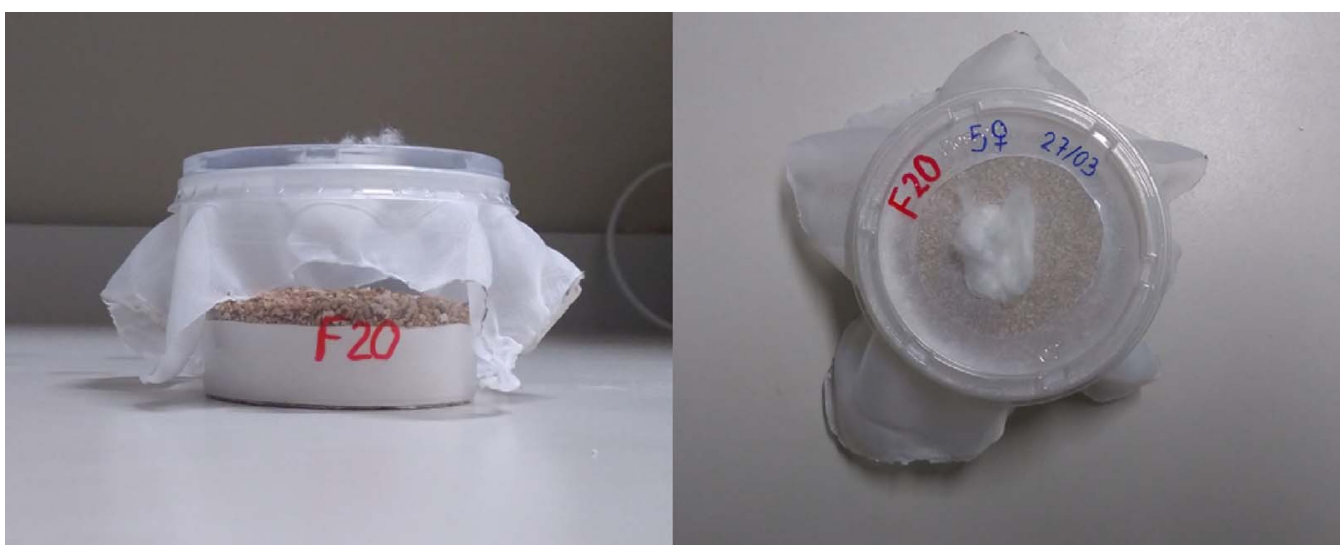

Fig. 2. Oviposition container with plaster of Paris at the bottom and vermiculite.

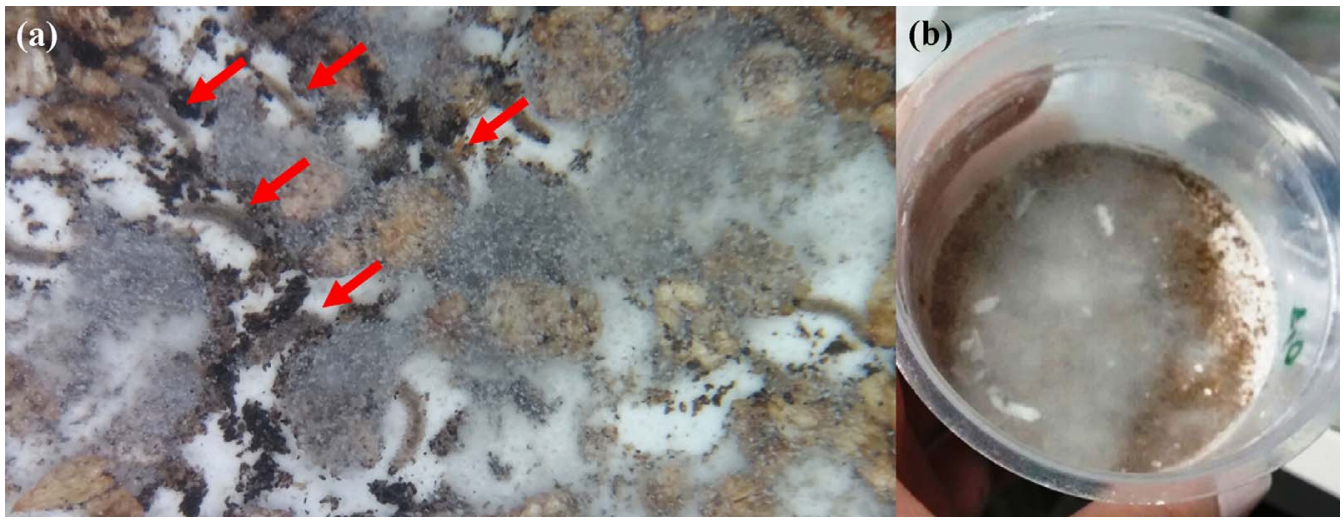

(a)

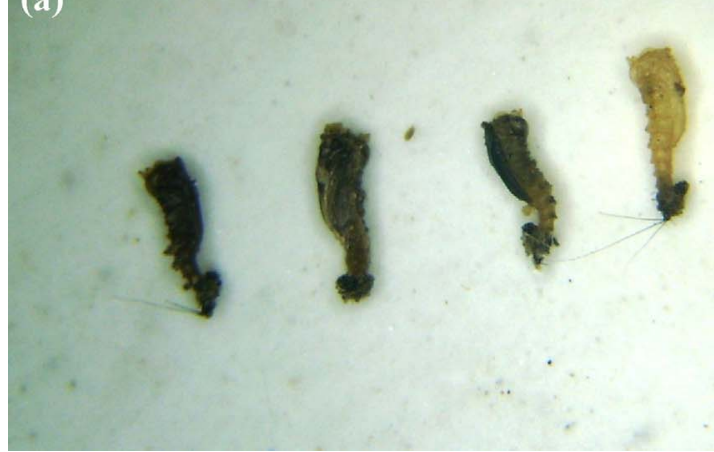

(b)

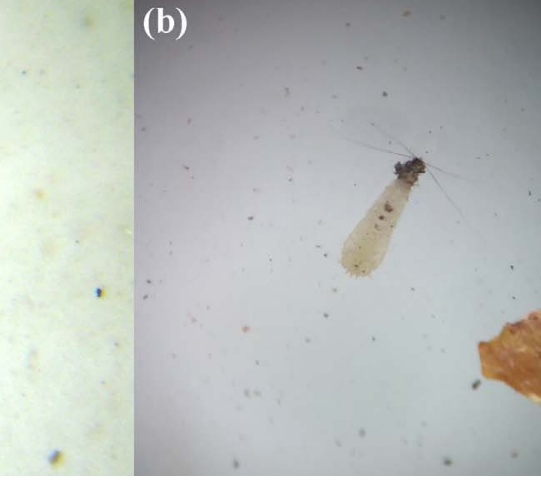

Fig. 3. Different types of fungi proliferation in the container (a) larvae (arrows) feeding on fungi, (b) fungi to be removed from the container.

Fig. 4. Sand fly pupae in different stages. (a) From left to right: two dead pupae, adult ready to emerge from the pupae, and normal healthy pupae; (b) newly formed pupae. 
humidity (80\%) must be placed in the Petri dishes. A piece of cotton wetted in 30\% sucrose solution is placed over the top of the cage, under the superior Petri dish (Fig. 1). Attention should be paid not to soak the cotton in the sucrose solution to the point of saturation since it can kill the insects by adherence.

This preparation avoids mortality of the insects during transportation from the field to the laboratory. It is important to have air-conditioning, an air humidifier, a wind curtain, and a controlled photoperiod provided in the breeding room of the insects.

\subsection{Blood feeding}

Our studies demonstrated BALB/c mice as good options for $\mathrm{Ny}$. neivai blood sources in laboratory conditions. Latex gloves must be avoided when manipulating animals or any colony material, since latex repels Ny. neivai (personal observation). Blood-feeding and colony maintenance should be carried out inside an air-conditioned room, $26 \pm 1{ }^{\circ} \mathrm{C}, 12: 12$ (L:D) photoperiod, and 80-90\% humidity. In our laboratory, several attempts to maintain $\mathrm{Ny}$. neivai in BOD incubators were made, however this species did not adapt to that environment.

The mouse should be weighed and the anaesthetic dosage calculated: Xylazine $2 \%(0.05 \mathrm{~mL} / 200 \mathrm{mg})$ and Ketamine $10 \%(0.20 \mathrm{~mL} /$ $200 \mathrm{mg}$ ). Anaesthetics are applied intramuscularly with a $1 \mathrm{~mL}$ short needle insulin syringe. This dose lasts for one hour. The mouse is then moved back to the maintenance mouse box and observed (Erhardt et al., 1984). The mouse should be placed in a Petri dish inside the cage to prevent urine and faeces touching the bottom tissue. In high insect density situations, two or three mice are required or the sand flies should be separated into more cages.

It has been noticed that female Ny. neivai which have fed on the same animal several times die before oviposition. Repeated bites evoke the production of antibodies by the host causing female mortality (Ghosh and Mukhopadhyay, 1998). However, further studies should evaluate the exact number of times the same animal can be used for blood feeding.

A second blood meal improved the number of adults in the colony. This procedure helps the maintenance and growth of $\mathrm{Ny}$. neivai insect numbers. Success in third blood meals is rare.

Regarding the routine of the Ny. neivai colony, between five and ten couples (blood-fed females and an equal number of males) is the ideal number of insects in each oviposition container (Goulart et al., 2015).

\subsubsection{Ethics statement}

The animal work was conducted according to the Guidelines for Animal Experimentation of the Conselho Nacional de Controle de Experimentação Animal (CONCEA). This study was approved by the Committee of Animal Experiments (CEUA) of the Universidade Estadual Paulista, UNESP, Araraquara (protocol number 54/2016).

\subsection{Oviposition and breeding containers}

A circle is cut at the bottom $(2.5 \mathrm{~cm}$ diameter) and in the lid $(4 \mathrm{~cm}$ diameter) of a $145 \mathrm{~mL}$ polystyrene container with thin curve tip scissors. Plaster of Paris is prepared as described by the manufacturer, and poured into the bottom of the container so the plaster leaks out of the circle, forming a thin layer of plaster on the outer bottom of the container. The container should be allowed to dry and cool and after one hour the process is complete.

A piece of voile tissue $(12 \times 12 \mathrm{~cm})$ should be cut and a small opening made at its center for transportation of the insects. The voile tissue is then placed together with the lid, closing the container. A piece of cotton is sufficient to close the hole at the center of the tissue, maintaining the insects inside the container (Fig. 2).

Field-caught sand flies are fed on mice and blood-fed females may be individualised, without males, in a rearing container with plaster of Paris at the bottom and $30 \%$ sucrose solution. The oviposition containers are moved to plastic boxes with two layers of wetted paper towel at the bottom. The plastic boxes are kept in drawers or cabinets to maintain the larvae in the dark through their life cycle to the adult phase. The layer of plaster of Paris on the containers touches the wetted paper towel of the plastic boxes, maintaining high humidity inside the oviposition containers.

Five days after oviposition the female is removed and specifically identified. Eggs of the same species are transferred to a $145 \mathrm{~mL}$ container (up to 300 eggs). A small amount of vermiculite should be added to the container (Fig. 2). A small piece of cotton wetted with $30 \%$ sucrose solution is placed over the voile lid and should be changed every $48 \mathrm{~h}$.

The following information can be written on the oviposition containers: date, insect generation, and number of blood-fed females.

For colony maintenance, after oviposition, live insects are transferred to a cage for a second blood meal and dead insects are removed with tweezers or box-cutters to avoid fungi proliferation. A round end brush is used to move the eggs on the walls of the containers to the bottom. Despite the high humidity, eggs laid on the walls of the container may desiccate.

\subsection{Larvae feeding}

An artificial diet is prepared with a 1:1:1:1 mix of rabbit faeces, rabbit food, fish food, and vegetable soil as described in Goulart et al. (2015). This mix is transferred to $15 \mathrm{~mL}$ Falcon tubes, autoclaved, and left to dry in a desiccation stove for a couple of hours. To assure that only fine powder will be offered to the larvae, a small piece of voile tissue is fixed with a rubber band to close the Falcon tube. The tube needs to be shaken to decompress the artificial diet. The tube should be closed with its own regular lid after the diet is utilised. The tubes can be autoclaved again after two or three months.

Larvae will start to emerge from the tenth day after oviposition, thus a small amount of artificial diet should be added on the ninth day.

\subsection{Humidity precautions}

Oviposition relies on humidity. Ideal humidity ranges from 70 to $80 \%$. After oviposition, the humidity should be reduced to $70 \%$ inside the plastic boxes. Droplet formation on the container walls can kill larvae and must be removed with a paper towel. The paper towels at the bottom of the organizer can be changed to further decrease humidity, or the cover of the organizer can be opened, for a short time, to dissipate the excess humidity.

\subsection{Fungi proliferation}

Several fungi species can appear inside the containers. In contaminated containers, it is worth moving the larvae to new, contaminant free containers. Alcohol 70\% wash solution, paper towels, and a cigarette lighter must be kept to sterilize the pin probes used on the containers contaminated with fungi.

Larvae can feed on some filamentous, cottony white fungal species that do not need to be removed (Fig. 3a). Pin probes should be used to mash the fungi and release larvae from their hyphae. The procedure must also be performed with pupae, as the emerged insect can be killed by the fungi hyphae (Fig. 3b).

Shaking the container lightly with the vermiculite helps to break down the fungi hyphae without damaging the larvae and pupae. This procedure must be avoided with first instar larvae due to their fragility.

Pathogenic microorganisms can promote mortality of larvae and pupae and these must be immediately removed. Containers presenting high larvae mortality should be washed in $70 \%$ alcohol and discarded.

The plastic boxes and paper towels on the bottom can become contaminated and must be washed or changed as necessary or at least once per week. 


\subsection{Pupation}

The development date of the first pupae should be noted on the lid of the container since it helps keep track of the colony and the next steps to be taken.

It is of great importance to learn how to identify pupae that are empty, whole, dead, newly formed, and ready to hatch. When adults are ready to emerge from the pupae the wing region becomes black (Fig. 4).

Newly formed pupae display white colour, changing to yellow-orange colour after a few hours which is maintained for at least seven more days (Fig. 4).

Empty containers should be cleaned with flat-end brushes and a sponge to remove dirt and then left to dry to be reutilized.

\subsection{Adult emergence}

Maintenance routine days are usually Monday, Wednesday, and Friday. On Fridays, a small piece of cotton wetted in 30\% sucrose solution is placed over the voile tissue of the containers with the presence of pupae to provide food for adults which will likely emerge on the same day or at the weekend.

The cotton should be removed on Mondays, as the newly emerged adults will be transferred to new cages during the week, properly identified with the insect generation and date of emergence. The cotton piece wetted in $30 \%$ sucrose solution, Petri dishes with cotton soaked in water, and the plastic bag wrapping the cage are essential to handle the adults of the colony. Both cotton and Petri dishes should be changed every week to avoid fungi proliferation.

Five to seven days after emergence, adults are ready for experimentation or blood feeding. Empty cages should be cleaned in three steps: i) immersion in chlorine bleach diluted in water (5\%); ii) observation of the removal of the dirt by the solution; iii) washing in running water.

\section{Competing interests}

The authors declare that they have no competing interests.

\section{Funding}

This work was supported by FAPESP (Fundação de Amparo à Pesquisa do Estado de São Paulo - Process2012/23832-9).

\section{Acknowledgements}

We would like to thank Dr. Danilo Ciccone Miguel for the first idea of the breeding protocol.

\section{References}

Andrade Filho, J.D., Galati, E.A.B., Falcão, A.L., 2004. Biology of the first generation of a laboratory colony of Nyssomyia intermedia (Lutz \& Neiva, 1912) and Nyssomyia neivai (Pinto, (Diptera: Psychodidae). Mem. Inst. Oswaldo Cruz 99 (6), 597-601.

Barretto, M.P., 1942. Contribuição para o estudo da biologia dos flebótomos em condições experimentais [dissertation]. Faculdade de Medicina da Universidade de São Paulo.

Elnaiem, D.E., Ward, R.D., 1991. Response of the sandfly Lutzomyia longipalpis to an oviposition pheromone associated with conspecific eggs. Med. Vet. Entomol. 5 (1), 87-91.

Elnaiem, D.E., Ward, R.D., 1992. Oviposition attractants and stimulants for the sandfly Lutzomyia longipalpis (Diptera: Psychodidae). J. Med. Entomol. 29 (1), 5-12.

Erhardt, W., Hebestedt, A., Aschenbrenner, G., Pichotka, B., Blümel, G., 1984. A comparative study with various anesthetics in mice (pentobarbitone, ketamine-xylazine, carfentanyl-etomidate). Res. Exp. Med. (Berl.) 184 (3), 159-169.

Ghosh, K.N., Mukhopadhyay, J., 1998. The effect of anti-sandfly saliva antibodies on Phlebotomus argentipes and Leishmania donovani. Int. J. Parasitol. 28 (2), 275-281.

Goulart, T.M., de Castro, C.F., Machado, V.E., da Rocha Silva, F.B., Pinto, M.C., 2015. Techniques to improve the maintenance of a laboratory colony of Nyssomyia neivai (Diptera: Psychodidae). Parasit Vectors 8, 423.

Killick-Kendrick, R., Killick-Kendrick, M., 1987. The laboratory colonization of Phlebotomus ariasi (Diptera: Psychodidae). Ann. Parasitol. Hum. Comp. 62 (4), 354-356.

Killick-Kendrick, M., Killick-Kendrick, R., 1991. The initial establishment of sandfly colonies. Parassitologia 33, 315-320.

Lawyer, P.G., Rowton, E.D., Perkins, P.V., Johnson, R.N., Young, D.G., 1991. Recent advances in laboratory mass rearing of phlebotomine sand flies. Parassitologia 361-364.

Meneses, C., Morales, E., Rhodes, C., Oliveira, F., Valenzuela, J.G., Kamhawi, S., 2014. CD: Sandfly Fellas. A Sandfly Rearing Guide [CD-ROM]. Rockville, Maryland, USA. Available from: https://www.vectorbase.org/forums/general-forums/methodsprotocols-reagents/cd-sand-fly-fellas-sand-fly-rearing-guide.

Modi, G.B., Tesh, R.B., 1983. A simple technique for mass rearing Lutzomyia longipalpis and Phlebotomus papatasi (Diptera: Psychodidae) in the laboratory. J. Med. Entomol 20 (5), 568-569.

Nieves, E., Ribeiro, A., Brazil, R., 1997. Physical factors influencing the oviposition of Lutzomyia migonei (Diptera: Psychodidae) in laboratory conditions. Mem. Inst. Oswaldo Cruz 92 (6), 733-737.

Rangel, E.F., Souza, N.A., Wermelinger, E.D., Barbosa, A.F., 1985. Estabelecimento de colônia, em laboratório, de Lutzomyia intermedia Lutz \& Neiva, 1912 (Diptera, Psychodidade, Phlebotominae). Mem. Inst. Oswaldo Cruz. 80 (2), 219-226.

Rangel, E.F., Souza, N.A., Wermelinger, E.D., Barbosa, A.F., Andrade, C.A., 1986. Biologia de Lutzomyia intermedia Lutz \& Neiva, 1912 e Lutzomyia longipalpis Lutz \& Neiva, 1912 (Diptera, Psychodidae), em condições experimentais. I. Aspectos da alimentação de larvas e adultos. Mem. Inst. Oswaldo Cruz. 81 (4), 431-438.

Santamaría, E., Munstermann, L.E., Ferro, C., 2002. Estimating carrying capacity in a newly colonized sand fly Lutzomyia serrana (Diptera: Psychodidae). J. Econ. Entomol. 95 (1), 149-154.

Souza, N.A., Andrade-Coelho, C.A., Vilela, M.L., Barbosa, A.F., Rangel, E.F., 1999. A new larval diet for colonization of Phlebotomine Sand flies. Mem. Inst. Oswaldo Cruz 94 (6), 845-847.

Volf, P., Volfova, V., 2011. Establisment and maintenance of sand fly colonies. J. Vector Ecol. 36 (Suppl. 1), S1-9.

Wermelinger, E.D., Zanuncio, J.C., 2001. Development of Lutzomyia intermedia and Lutzomyia longipalpis (Diptera: Psychodidae: Phlebotominae) larvae in different diets. Braz. J. Biol. 61 (3), 405-408.

Young, D.G., Perkins, P.V., Endris, R.G., 1981. A larval diet for rearing phlebotomine sand flies (Diptera: Psychodidae). J. Med. Entomol. 18 (5) 446-446. 